\title{
A RESULT ON THE CONVOLUTION OF DISTRIBUTIONS
}

\author{
by B. FISHER
}

(Received 14th June 1974)

\section{Introduction}

In a recent paper (1), Jones extended the definition of the convolution of two distributions to cover certain pairs of distributions which could not be convolved in the sense of the previous definition. The convolution $\omega_{1} * \omega_{2}$ of two distributions $\omega_{1}$ and $\omega_{2}$ was defined as the limit of the sequence $\omega_{1 n} * \omega_{2 n}$, provided the limit $\omega$ exists in the sense that

$$
\lim _{n \rightarrow \infty} \int_{-\infty}^{\infty} \phi(x) \omega_{1 n} * \omega_{2 n}(x) d x=\int_{-\infty}^{\infty} \phi(x) \omega(x) d x
$$

for all fine functions $\phi$ in the terminology of Jones (2) where

$$
\omega_{1 n}(x)=\omega_{1}(x) \tau(x / n), \quad \omega_{2 n}(x)=\omega_{2}(x) \tau(x / n)
$$

and $\tau$ is an infinitely differentiable function satisfying the following conditions:

(i) $\tau(x)=\tau(-x)$,

(ii) $0 \leqq \tau(x) \leqq 1$,

(iii) $\tau(x)=1$ for $|x| \leqq 1 / 2$,

(iv) $\tau(x)=0$ for $|x| \geqq 1$.

In (1) Jones proved that

$$
1 * \operatorname{sgn} x=x
$$

In the following we give a generalisation of this result.

\section{Result}

We prove that in the sense of Jones' definition of convolution for distributions

$$
x^{r} *\left(\operatorname{sgn} x \cdot x^{r}\right)=\frac{(r !)^{2}}{(2 r+1) !} x^{2 r+1}
$$

for $r=0,1,2, \ldots$, the particular case $r=0$ being equation (1).

We shall write

$$
\left(x^{r}\right)_{n}=x^{r} \tau(x / n), \quad\left(\operatorname{sgn} x \cdot x^{r}\right)_{n}=\left(\operatorname{sgn} x \cdot x^{r}\right) \tau(x / n)
$$


and so

$$
\begin{aligned}
\left(x^{r}\right)_{n} *\left(\operatorname{sgn} x \cdot x^{r}\right)_{n} & =\int_{-n}^{n}(x-t)^{r} \tau\left(\frac{x-t}{n}\right) t^{r} \tau(t / n) \operatorname{sgn} t d t \\
& =\int_{0}^{n}\left\{(x-t)^{r} \tau\left(\frac{x-t}{n}\right)-(-1)^{r}(x+t)^{r} \tau\left(\frac{x+t}{n}\right)\right\} t^{r} \tau(t / n) d t \\
& =(-1)^{r} n^{2 r+1} \int_{0}^{1}\left\{f\left(t-\frac{x}{n}\right)-f\left(t+\frac{x}{n}\right)\right\} f(t) d t,
\end{aligned}
$$

where

$$
f(t)=t^{r} \tau(t)
$$

It follows that

$\left(x^{r}\right)_{n} *\left(\operatorname{sgn} x \cdot x^{r}\right)_{n}=-2(-1)^{r} n^{2 r+1} \sum_{s=1}^{r+1} \frac{x^{2 s-1}}{(2 s-1) ! n^{2 s-1}} \int_{0}^{1} f^{(2 s-1)}(t) f(t) d t+R_{n}(x)$ where for each bounded closed interval $I$, there is a constant $C$ (depending on $I$ ) such that

$$
\left|R_{n}(x)\right| \leqq \frac{C}{n} \text { for all } x \in I .
$$

Then for all fine functions $\phi$ vanishing outside $I$

as $n$ tends to $\infty$.

$$
\left|\int_{-\infty}^{\infty} R_{n}(x) \phi(x) d x\right| \leqq \frac{C}{n} \int_{I}|\phi(x)| d x \rightarrow 0
$$

Now since

it follows that

$$
\begin{gathered}
f^{(r)}(0)=r ! \\
f(0)=f^{\prime}(0)=\ldots=f^{(r-1)}(0)=0, \\
f(1)=f^{\prime}(1)=\ldots=f^{(r)}(1)=0,
\end{gathered}
$$

$$
\int_{0}^{1} f^{(2 s-1)}(t) f(t) d t=(-1)^{s} \int_{0}^{1} f^{(s-1)}(t) f^{(s)}(t) d t
$$

$$
=0
$$

for $s=1,2, \ldots, r$ and

Thus

$$
\begin{aligned}
\int_{0}^{1} f^{(2 r+1)}(t) f(t) d t & =(-1)^{r} \int_{0}^{1} f^{(r)}(t) f^{(r+1)}(t) d t \\
& =-\frac{1}{2}(-1)^{r}(r !)^{2}
\end{aligned}
$$

$$
\left(x^{r}\right)_{n} *\left(\operatorname{sgn} x \cdot x^{r}\right)_{n}=\frac{(r !)^{2}}{(2 r+1) !} x^{2 r+1}+R_{n}(x)
$$


and so for an arbitrary fine function $\phi$ we have

$$
\lim _{n \rightarrow \infty} \int_{-\infty}^{\infty} \phi(x)\left(x^{r}\right)_{n} *\left(\operatorname{sgn} x \cdot x^{r}\right)_{n} d x=\frac{(r !)^{2}}{(2 r+1) !} \int_{-\infty}^{\infty} x^{2 r+1} \phi(x) d x,
$$

since a fine function vanishes identically outside a finite interval. This completes the proof of equation (2).

\section{REFERENCES}

(1) D. S. Jones, The convolution of generalized functions, Quart. J. of Math. Oxford Ser. (2) 24 (1973), 145-163.

(2) D. S. JONES, Generalized functions (McGraw-Hill, 1966).

\section{UNIVERSITY OF LEICESTER}

